\title{
Cloning and Expression of a Novel Target Fusion Protein and its Application in Anti- Tumor Therapy
}

\author{
Rui Sun ${ }^{\mathrm{a}}$ Yan Zhu Hailiang Feng $^{\mathrm{b}}$ Zhenli Yang $^{\mathrm{b}}$ Xiaocui Bian ${ }^{\mathrm{b}}$ Pei Gu \\ Chunjing Wang ${ }^{b}$ Yuqin Liua,b
}

\begin{abstract}
aDepartment of Pathology, Institute of Basic Medical Sciences, Chinese Academy of Medical Sciences and Peking Union Medical College, Tsinghua University, Beijing, ${ }^{b} C e l l$ Resource Center, Institute of Basic Medical Sciences, Chinese Academy of Medical Sciences and Peking Union Medical College, Tsinghua University, Beijing, China
\end{abstract}

\section{Key Words}

EGF • Recombinant protein • Immunodominant epitopes $•$ Listeriolysin O • Anti-tumor therapy

\begin{abstract}
Backgrounds: Epidermal growth factor (EGF) is a 53 amino acid polypeptide and its receptor EGFR is an established therapeutic target for anti-tumor therapy. Two major categories of EGFR-targeted drugs include monoclonal antibodies (mAbs) and small molecular tyrosine kinase inhibitors (TKIs). However, drug resistance occurs in a significant proportion of patients due to EGFR mutations. Since EGFR can maintain activation while abrogating the activity of mAbs or TKIs, or bypass signaling functions while successfully circumventing the EGFEGFR switch, developing new mechanism-based inhibitors is necessary. Methods: In this study, based on the principle of tumor immunotherapy, a recombinant protein pLLO-hEGF was constructed. The $\mathrm{N}$-terminal portion contains three immunodominant epitopes from listeriolysin O (LLO) and the C-terminal includes EGF. To use EGF as a target vector to recognize EGFR-expressing cancer cells, immunodominant epitopes could enhance immunogenicity of tumor cells for immune cell activation and attack. Results: Recombinant protein pLLO-hEGF was successfully expressed and showed strong affinity to cancer cells. Also, pLLO-hEGF could significantly stimulate human lymphocyte proliferation and the lymphocytes demonstrated enhanced killing potency in EGFR-expressing cancer cells in vitro and in vivo. Conclusion: This study can provide novel strategies and directions in tumor biotherapy.
\end{abstract}

Copyright (C) 2014 S. Karger AG, Basel

Yuqin Liu

KARGER 125
Institute of Basic Medical Sciences, Chinese Academy of Medical Sciences and Peking

Union Medical College, Tsinghua University, \#5, Dong Dan San Tiao, Dongcheng District, Beijing 100005 (China)

Tel. +86 010 69156473, Fax +86 01069156473 , E-Mail ccc5@ibms.pumc.edu.cn 


\section{Introduction}

So far, a collection of solid malignancies is marked by EGFR overexpression including breast [1], prostate [2], kidney [3], bladder [4], ovary [5], colon [6], brain [7], lung [8], head and neck [9] and pancreatic cancers [10]. EGFR has been selected as a crucial target for anticancer therapy. Traditional EGFR-targeted drugs include monoclonal antibodies (mAbs) and small-molecule tyrosine kinase inhibitors (TKIs). However, recent reports showed that almost all patients with metastatic lung cancer and other tumor types that initially benefit from EGFR-targeted therapies eventually develop resistance [11]. The mechanisms of tumor tolerance to EGFR inhibitors involve the cross-talk between different oncogenic signal pathways and the "loss of function" mutation of EGFR $[12,13]$. EGFR-activating mutations that target the kinase catalytic domain abrogate autoinhibition and result in significant increase in kinase activity [14]. In either "increased activity" or "loss of function" case, the role of EGF interacting with EGFR during the oncogenic signaling transduction is weakened and even abolished. Thus, the elucidated molecular mechanism of primary and acquired resistance to EGFR-targeted therapy will contribute to develop novel anti-EGFR agents.

In recent decades, the use of bacteria-derived components to develop anti-tumor agents has made significant advance in tumor immunotherapy. To achieve successful tumor immunotherapy, breaking immune tolerance and eliciting a strong immune response to tumor antigens are critical since most tumor-associated antigens (TAAs) are low immunogenic [15]. Previous studies suggested that bacteria-derived molecules could be used to surmount immune unresponsiveness to TAAs. In fact, the application of bacteria or bacteria-derived components as non-specific immunostimulatory agents in anti-tumor therapy, can be traced back 100 years ago when Coley' toxins were created to cure a malignant tumor [16].

In the present study, we first created a recombinant gene encoding an immunogenic target-peptide by fusing human EGF with three immunodominant epitopes derived from listeriolysin 0 (LLO) secreted by Listeria monocytogenes $(\mathrm{Lm})$. Three immunodominant epitopes were designed on the $\mathrm{N}$-terminal and EGF on the C-terminal. This recombinant protein can be used in tumor immunotherapy by mimicking native antigens on the tumor cell surface through its "EGF tail" coupled to EGFR, on the premise that for these tumors, EGF-EGFR coupling cannot accelerate oncogenic signal transduction and promote cancer cell proliferation. $\mathrm{Lm}$ is a facultative intracellular microorganism that can enter and multiply in a wide variety of eukaryotic cells. LLO plays a critical part in mediating $\mathrm{Lm}$ phagosomal escape and facilitating multiplication during bacterial infection [17]. LLO contains abundant $\mathrm{CD}^{+}$and $\mathrm{CD}^{+} \mathrm{T}$ cells epitopes and three immunodominant epitopes have been determined: a dominant $\mathrm{CD}^{+} \mathrm{T}$ cell epitope, $\mathrm{LLO}_{91-99}$ (residues 91-99) [18], and two typical CD4 ${ }^{+} \mathrm{T}$ cell epitopes, $\mathrm{LLO}_{189-201}$ (residues 189-201) [19], and $\mathrm{LLO}_{215-226}$ [20]. T lymphocytes with T cell receptor (TCR) recognizing these peptides are easily amplified from the $\mathrm{T}$ cell pool. In this study, we designed and generated the recombinant protein pLLO-hEGF by linking these immunodominant epitopes with EGF, and preliminarily investigated its potency in tumor immunotherapy.

\section{Materials and Methods}

Strains and reagents

Escherichia coli BL21 (DE3) strain, UPMC 17 in WFCC Global Catalogue of Microorganisms and its genotype of F-ompT gal dcm lon hsdSB(rB mB') $\lambda$ (DE3 [lacl lacUV5-T7 gene 1 ind1 sam7 nin5]), was purchased from TransGen Biotech (Beijing, China). The expression vector pET-30a(+) was purchased from Novagen (Germany). Primers and recombinant gene A-336 (pLLO-hEGF) were synthesized by Invitrogen (Germany). Restriction enzymes BamH I and Xho I were purchased from New England Biolabs (USA). Other reagents were obtained from standard commercial sources and were of analytical reagent grade. 
Mice, cancer cell lines, and human peripheral blood mononuclear cell (PBMC)

Male BALB/c nu/nu mice (License Number: SCXK 2009-0017) of 6 8 weeks of age were purchased from National Institutes for Food and Drug Control (Beijing, China), and all mice were kept under specific pathogen-free conditions. All experimental procedures involving animals were approved by the Institutional Animal Care and Use Committee (IACUC) of Institute of Basic Medical Sciences (Beijing, China). All human cancer cell lines used in this study were from Cell Resource Center, Institute of Basic Medical Sciences, Chinese Academy of Medical Sciences and Peking Union Medical College (Beijing, China) and have been authenticated by Short Tandem Repeat (STR) profiling (http://www.cellresource.cn). Cells were cultured as described in the product protocols. HCT116 and HT-29 human colorectal carcinoma cells were separately maintained in IMDM medium and IMDM/F-12(1:1) medium containing 5\% fetal bovine serum (FBS) (Gibco-BRL, USA). A549 and NCI-H157 human non-small-cell lung cancer cells were cultured in McCoy' 5A medium and RPMI 1640 medium supplemented with 10\% FBS, respectively. SK-BR-3 and MDA-MB-231 human breast cancer cells were separately cultured in RPMI 1640 medium and Leibovitz's L-15 medium including 10\% FBS. Fresh blood collected from different healthy volunteers was supplied by Beijing Red Cross Blood Center and approved by Medical Ethics Committee of Peking Union Medical College, and the isolation of human peripheral blood mononuclear cells (PBMCs) was performed according to density gradient centrifugation by LSM ${ }^{\circledR}$ Lymphocyte Separation Medium (50494, MP Biomedicals, USA). Briefly, 3 $\mathrm{ml}$ of LSM was aseptically transferred into a $15 \mathrm{ml}$ centrifuge tube and thoroughly mixed by gently inverting the tube. Next, $2 \mathrm{ml}$ of the anti-coagulated blood was diluted with an equal volume of physiological saline and carefully layered over $3 \mathrm{ml}$ of LSM to create a sharp blood-LSM interface. After centrifugation at room temperature with a speed of $400 \mathrm{~g}$ for $30 \mathrm{~min}$, buffy coat was collected, and washed twice with PBS. Finally, human PBMCs were resuspended in RPMI 1640 medium supplemented with $2 \mathrm{mM} \mathrm{L-glutamine,} 100 \mathrm{U} / \mathrm{ml}$ penicillin G sodium, $100 \mu \mathrm{g} / \mathrm{ml}$ streptomycin sulphate and 200U/ml rhIL-2 (PeproTech Inc., USA). All cells were cultured in a humidified incubator with $5 \% \mathrm{CO}_{2}$ at $37^{\circ} \mathrm{C}$.

\section{Design and molecular cloning of $p L L O-h E G F$}

The recombinant gene was composed of coding sequences of three immunodominant epitopes, $\mathrm{LLO}_{91-}$ ${ }_{99} \mathrm{LLO}_{189-201}$ and $\mathrm{LLO}_{215-226}$, the linker "GSGGSG", and human EGF, in the following order: $\mathrm{LLO}_{91-99}$ - linker - $\mathrm{LLO}_{189-201}$ - linker - $\mathrm{LLO}_{215-226}$ - linker - human EGF. Since the N-end composition of LLO is important in the ubiquitin-proteasome degradation pathway so that the CD8 ${ }^{+} \mathrm{T}$ cell epitope $\mathrm{LLO}_{91-99}$ can be processed and presented by MHC class I molecules [17, 21, 22], six additional amino acids flanking $\mathrm{LLO}_{91-99}$ were reserved, with "RK" on the left and "VVEK" on the right, as charged or polar residues. Therefore, the primary structure of fusion peptide (111 amino acids) is as follows: N-end - RK - GYKDGNEYI - VVEK - GSGGSG - WNEKYAQAYPNVS GSGGSG - SQLIAKFGTAFK - GSGGSG - NSDSECPLSHDGYCLHDGVCMYIEALDKYACNCVVGYIG ERCQYRDLKWWELR-C-end. The corresponding DNA sequence was synthesized artificially as gene A-336 (pLLO-hEGF) and amplified by PCR using specific primers (forward 5' - GCG GAT CCA GAA AAG GTT AC - $3^{\prime}$ with an in-frame BamH I restriction site, and reverse $5^{\prime}$ - GCT CGA GTT AGC GCA GTT C - $3^{\prime}$ with an in-frame Xho I restriction site). PCRs were performed as follows: initial denaturation at $94^{\circ} \mathrm{C}$ for $5 \mathrm{~min}$ followed by 35 cycles at $94^{\circ} \mathrm{C} / 30 \mathrm{~s}, 46^{\circ} \mathrm{C} / 30 \mathrm{~s}$, and $72^{\circ} \mathrm{C} / 45 \mathrm{~s}$; and final extension was at $72^{\circ} \mathrm{C} / 7 \mathrm{~min}$. The amplified DNA products were cloned into the pET-30a(+) expression vectors.

\section{Expression and purification of pLLO-hEGF}

The recombinant plasmid pET-30a(+)-pLLO-hEGF was transformed into E. coli BL21 (DE3) and confirmed by DNA sequencing. The expression of recombinant pLLO-hEGF was induced by isopropyl-betaD-thiogalactoside (IPTG) when the absorbance at $600 \mathrm{~nm}\left(A_{600}\right)$ of $E$. coli grown in LB-medium containing kanamycin reached 0.6. Two forms of pLLO-hEGF were expressed as a (His) ${ }_{6}$-tagged protein existing under different inducing conditions: one was inclusion bodies induced by $1.0 \mathrm{mM} \mathrm{IPTG}$ at $37^{\circ} \mathrm{C}$ for $4 \mathrm{~h}$; and the other was soluble protein induced by $0.5 \mathrm{mM} \mathrm{IPTG}$ at $23^{\circ} \mathrm{C}$ for $8-10 \mathrm{~h}$ with the maximum of soluble expression. After low-temperature induction, the pellets were harvested, resuspended and ultrasonicated. Supernatants were acquired by centrifugation $\left(12,000 \times \mathrm{g}, 4^{\circ} \mathrm{C}, 40 \mathrm{~min}\right)$. Soluble pLLO-hEGF was purified from supernatants and the (His) $6_{6}$ tag was removed using thrombin-cleaving under native conditions by $\mathrm{Co}^{2+}$-based TALON Metal Affinity Resins Purification Kit (Clontech Laboratories, Inc.). Recombinant pLLOhEGF was identified by $12 \%$ sodium dodecyl sulfate polyacrylamide gel electrophoresis (SDS-PAGE) and western blot analysis using ant-(His) ${ }_{6}$ and anti-EGF mAbs. Extracted proteins were filtered through a 0.22 
$\mu \mathrm{m}$ Supor $^{\circledR}$ Membrane (Pall Corporation, USA) to eliminate bacterial pollution, and refined by removing endotoxin lipopolysaccharide (LPS) through ToxinEraser Endotoxin Removal Kit (GenScript USA Inc.). Purified pLLO-hEGF was stored at $-20^{\circ} \mathrm{C}$ for further studies.

\section{EGFR expression in human cancer cell lines}

Expression levels of EGFR in 21 human cancer cell lines were detected by western blot analysis. Human cancer cell lines in this study included human breast cancer cell lines (MDA-MB-231, MDA-MB-453, MCF7, SK-BR-3), human lung cancer cell lines (NCI-H460, NCI-H661, NCI-H1703, NCI-H2170, NCI-H1650, NCI-H157, A549), and human colorectal cancer cell lines (HCT116, HT-29, HCT-8, SW620, LoVo, COLO201, Caco-2, COL0205, COLO320DM, SW480). Total proteins of cultured cancer cells were extracted using protein lysis buffer supplemented with phenylmethylsulfonyl fluoride (PMSF) and protease inhibitors (Amresco, USA). Protein samples were separated by 12\% SDS-PAGE and transferred onto nitrocellulose (NC) membranes (Pall Gelman Laboratory, England). After blocking with 5\% non-fat milk at room temperature for $2 \mathrm{~h}$, followed by three washes with TBST solution $(0.01 \mathrm{M}$ Tris-buffered saline containing $0.05 \%$ Tween 20, pH 7.4), NC membranes were incubated with primary antibodies overnight at $4^{\circ} \mathrm{C}$, including anti-EGFR rabbit mAb (Cell Signaling Technology, USA), and anti-GAPDH mouse mAb (Santa Cruz, USA) and anti- $\beta$ Tubulin rabbit PcAb (Beijing Cowin Biotech, China) as internal controls. HRP-conjugated goat anti-rabbit or anti-mouse IgG (Zhongshan Golden-bridge Biotechnology, China) reacted with primary antibodies at room temperature for $2 \mathrm{~h}$. After three washes with TBST, immunoreactive bands were visualized using SuperSignal West Pico Chemiluminescent Substrate (Thermo Fisher Scientific, USA).

\section{Cell proliferation assay}

Six cell lines with high EGFR expression were analyzed: HCT116, HT-29, A549, NCI-H157, MDAMB-231 and SK-BR-3. The effects of pLLO-hEGF on proliferation of these cancer cells were detected by the MTT method [23]. In brief, according to different growth rates cancer cells were seeded into 96-well plates (Corning Inc, USA) at different densities (HCT116, 1,000 cells/well; HT-29, 2,000 cells/well; A549, 1,000 cells/well; NCI-H157, 2,000 cells/well; SK-BR-3, 2,500 cells/well; MDA-MB-231, 3,000 cells/well) in a final volume of $100 \mu \mathrm{L}$ medium. In vivo, EGF concentrations in serum can be between $(0.1-2) \times 10^{-9} \mathrm{M}$, with local tissue concentrations as high as $\sim 2 \times 10^{-8} \mathrm{M}[24,25]$. Therefore, concentration gradients of pLLO-hEGF from low to high were set as follows: $10^{-10} \mathrm{M} ; 10^{-9} \mathrm{M} ; 10^{-8} \mathrm{M} ; 10^{-7} \mathrm{M}$, EGF was used as parallel controls and PBS as blank controls. After 24 h, pLLO-hEGF ( 6 repeated wells) and EGF ( 6 repeated wells) were added. Then, at 48, 72, 96 and $120 \mathrm{~h}$, MTT (Sigma, USA) was added to each well at a final concentration of $0.5 \mathrm{mg} / \mathrm{ml}$, followed by culture at $37^{\circ} \mathrm{C}$ for $4 \mathrm{~h}$. Finally, the supernatant of each well was removed by a vacuum pump and $100 \mu \mathrm{l}$ DMSO was added to dissolve blue formazan crystals. The absorbance of each well at $570 \mathrm{~nm}\left(A_{570}\right)$ was detected on a Synergy ${ }^{\mathrm{TM}} 4$ reader (BioTek Instruments, Inc.).

\section{Cell immunofluorescent staining}

Immunocytochemistry assay was used to analyze if pLLO-hEGF could bind to EGFR-expressing cancer cells. EGF and anti-EGFR mAb (Cell Signaling Technology, USA) were parallel controls and PBS was used as the blank control. Cancer cells (HCT116, HT-29, A549, NCI-H157, MDA-MB-231 and SK-BR-3) were cultured on cover slides and fixed using 4\% paraformaldehyde (PFA) according to an improved method as previously described [26]. After the slides were washed twice in $3 \mathrm{ml}$ PBS for $5 \mathrm{~min}$ each at room temperature, the anti-EGFR mAb group was directly incubated with anti-EGFR rabbit mAb (the primary antibody) overnight at $4^{\circ} \mathrm{C}$, while EGF and pLLO-hEGF groups were incubated with EGF and pLLO-hEGF, respectively, for $8 \mathrm{~h}$ at $4^{\circ} \mathrm{C}$, followed by anti-EGF mouse mAb (Beijing Hapten and Protein Biomedical Institute, China) (the primary antibody) overnight at $4^{\circ} \mathrm{C}$. The PBS group was incubated with PBS. Slides were then rinsed and incubated with FITC-labeled goat anti-rabbit or anti-mouse IgG (Zhongshan Golden-bridge Biotechnology, China) (the secondary antibody) at room temperature for $50 \mathrm{~min}$. After three washes, the slides were counterstained by mounting medium with DAPI (Sigma, USA) and micrographs were captured with Olympus IX51 $(20 \times 10$ amplification).

\section{Human PBMC proliferative response to pLLO-hEGF}

Lymphocyte proliferation assay was performed to analyze if pLLO-hEGF could stimulate $\mathrm{T}$ cells proliferation. A long culture period of 14 days in vitro was used since a normal primary immune response by antigen stimulation will take approximately 7 days or longer. Human PBMCs were cultured in complete 
RPMI 1640 medium (200U/ml rhIL-2 added to keep T cell survival, but not proliferation), and seeded into 24-well plates at a density of $2 \times 10^{6}$ cells/well in a final volume of $1.0 \mathrm{ml}$. PBMCs from the same person were divided into three groups based on different stimuli: PBS (negative control), pLLO-hEGF (10 $\mu \mathrm{g} / \mathrm{ml}$ ) and Con A ( $5 \mu \mathrm{g} / \mathrm{ml}$, positive control) (Sigma, USA). The concentration of pLLO-hEGF was not very high, but to maintain its effect, pLLO-hEGF was supplemented when lymphocytes received fresh medium. On day 3,7 and 14, lymphocytes of each group were separately harvested and the absolute number of cells was calculated by Scepter $^{\mathrm{TM}}$ 2.0 Handheld Automatic Cell Counter (Millipore Corporation, USA). Then, $1.0 \times 10^{5}$ cells from each group of each person were subjected to phenotype analysis by surface staining using CD3FITC, CD4-APC and CD8-PerCP mAbs (BD Pharmingen ${ }^{T M}$, USA) according to the manufacturer's instructions. The labeled lymphocytes were examined on a flow cytometer (Accuri ${ }^{\mathrm{TM}} \mathrm{C} 6, \mathrm{BD}$ Biosciences) and data were analyzed on BD Accuri C6 software. The absolute number of each type of T cell was calculated through cell total number and cell percentage. This assay was repeated three times based on different lymphocyte sources provided by different people.

Cytotoxicity assay in vitro

MTT-based cytotoxicity assays were performed using HCT116, HT-29, A549, NCI-H157, and SKBR-3 cancer cells according to a modified method previously described [27]. To verify that lymphocytes stimulated by pLLO-hEGF could kill EGFR-expressing cancer cells with pLLO-hEGF helping in tumor cells recognition, the assay was slightly changed as follows: first, cancer cells were seeded into 96-well plates for $3 \mathrm{~h}$, and pLLO-hEGF was added to bind to cancer cells via the "EGF tail"; the cells were washed using PBS to remove extra pLLO-hEGF in medium, and the stimulated lymphocytes (14 days) were added into 96-well plates in a 50:1 ratio of effector:target (E:T). Lymphocytes stimulated with PBS and Con A were treated the same. After $12 \mathrm{~h}$, MTT (Sigma, USA) was added and cells were incubated at $37^{\circ} \mathrm{C}$ for $4 \mathrm{~h}$. Supernatants were carefully removed after centrifugation at a speed of 2,000 rpm for $10 \mathrm{~min}$, and $100 \mu \mathrm{L}$ DMSO was added to dissolve formazan crystals. The absorbance of each well at $570 \mathrm{~nm}\left(A_{570}\right)$ was detected by an automated microplate reader (Bio-Rad, USA), and the specific killing rates were expressed as $\left[1\right.$ - ( $A_{570 \text { (experimental group) }}$ - $\left.\left.A_{570 \text { (lymphocyte group) }}\right) / A_{570 \text { (cancer cell group) }}\right] \times 100 \%$. This assay was based on the assumption that lymphocytes recognizing pLLO-hEGF would recognize cancer cells with pLLO-hEGF binding, and show cytotoxicity. Three replications were performed.

Anti-tumor activity of lymphocytes stimulated by $p L L O-h E G F$ in vivo

A BALB/c-nude mouse model of human colorectal cancer (cell line HCT116) was established to evaluate the efficacy of lymphocytes stimulated by pLLO-hEGF to inhibit tumor growth. This protocol was previously established in several nude mice models. The nude mice were randomly divided into five groups as follows: Group A, treated with PBS as negative control; Group B, treated early with lymphocytes primed by pLLO-hEGF, and pLLO-hEGF; Group C, treated early with lymphocytes stimulated by PBS; Group D, treated late with lymphocytes primed by pLLO-hEGF, and pLLO-hEGF; Group E, treated late with lymphocytes stimulated by PBS. HCT116 cells were harvested during exponential growth and resuspended with PBS. On day 1 , each mouse was subcutaneously injected with $2 \times 10^{6}$ HCT116 cells in the left flank of nude mice. On day 2, each mouse of Group B was injected with $2 \times 10^{6}$ lymphocytes primed by pLLO-hEGF and $2.0 \mathrm{mg} / \mathrm{kg}$ pLLO-hEGF in peritumoral areas. Group C was injected with $2 \times 10^{6}$ lymphocytes stimulated by PBS. Group A was injected with $100 \mu \mathrm{L}$ PBS. On day 8, Group D and Group E were treated identically with Group B and Group C, respectively. At an interval of 7 days, the above groups received the same treatment. Except for Group A, every group was infused with lymphocytes three times. Tumor size was measured twice per week using a vernier caliper, and tumor volume $\left(\mathrm{cm}^{3}\right)$ was calculated using the formula: length $\times$ width $\times$ width $/ 2$. Body weight was recorded twice a week. Mice were sacrificed when tumor diameter reached $1.0 \mathrm{~cm}$, and the blood of each group was collected. PBMCs were isolated from blood for detecting the existence of human lymphocytes using anti-human CD3-FITC mAb by flow cytometry. Tumor masses were aseptically removed and weighed, and parts were fixed into 10\% buffered-formalin and paraffin-embedded for hematoxylin and eosin (HE) staining and immunohistochemistry analysis.

\section{Immunohistochemistry}

The procedure was performed using previously described methods [28]. Briefly, sections were deparaffinized with xylene and rehydrated in a series of decreasing concentrations of ethanol solutions. After heat-induced antigen retrieval and blocking endogenous peroxidase, the slides of tissue were incubated 
with mouse anti-human CD3 mAb (Zhongshan Golden-bridge Biotechnology, China) at room temperature for $2 \mathrm{~h}$. Then, after washing with PBS twice, the slides were incubated with HRP-labeled goat anti-mouse IgG secondary antibody at room temperature for $2 \mathrm{~h}$. Finally, a DAB Detection Kit was used to chromogenic reaction for visualization.

\section{Statistical analysis}

The data are represented as the mean \pm SEM. Statistical analyses were performed by one-way analysis of variance (ANOVA) with modified Bonferroni post hoc test. $P<0.05$ was considered a significant difference. For figures, $\left.{ }_{*}\right)$ denotes $P<0.05$. The software SPSS version 16.0 was used to data processing.

\section{GenBank accession numbers}

The accession number of recombinant protein pLLO-hEGF is KM888321, the accession number of listeriolysin O (LLO) is DQ054588.1, and Homo sapiens EGF is NM_001963.4.

\section{Results}

Cloning, expression, purification and identification of pLLO-hEGF

Gene A-336, named pLLO-hEGF and artificially designed, was successfully cloned into the pET-30a(+) expression vector and expressed as a (His) ${ }_{6}$-tagged protein in E. coli BL21 (DE3) after induction by IPTG (Fig. 1A). When induced by $1.0 \mathrm{mM}$ IPTG at $37^{\circ} \mathrm{C}$ for $4 \mathrm{~h}, \mathrm{pLLO}$-hEGF was overexpressed in the form of inclusion bodies and showed a single band in 12\% SDS-PAGE with an expected molecular mass of $16 \mathrm{kDa}$ (Fig. 1B). Soluble pLLO-hEGF was acquired by induction at $23^{\circ} \mathrm{C}$ with $0.5 \mathrm{mM}$ IPTG for $8-10 \mathrm{~h}$, and purified by $\mathrm{Co}^{2+}$-based affinity chromatography and thrombin-cleavage of $6 \times$ His tag under native conditions using non-denaturation reagent (1X Equilibration/Wash buffer ( $\mathrm{pH} 7.0$ ), $50 \mathrm{mM}$ sodium phosphate, $300 \mathrm{mM} \mathrm{NaCl}$ (Fig. 1C). Recombinant protein pLLO-hEGF was verified by western blot analysis using anti-(His) ${ }_{6}$ and anti-EGF mAbs. When the $6 \times$ His tag was not removed by thrombin-cleavage, pLLO-hEGF could be successfully identified by anti-(His) $\mathrm{mAb}$ and while the $6 \times$ His tag was removed, pLLO-hEGF could still be identified by anti-EGF mAb (Fig. 1D).

\section{The expression of EGFR in human cancer cell lines}

The expression of EGFR in different cancer cell lines was studied by western blot analysis (Fig. 2). Among human breast cancer cell lines, MDA-MB-231 and SK-BR-3 showed a high level of EGFR expression, MCF7 expressed small levels and MDA-MB-453 did not show any expression, while GAPDH was used as an internal control (Fig. 2A). Among human lung cancer cell lines, only NCI-H2170 did not express EGFR (Fig. 2B). In addition, except for SW620 and COLO320DM, all human colorectal cancer cells expressed EGFR (Fig. 2C). Previous studies showed that EGFR is expressed or highly expressed in a range of solid tumors including breast, head and neck, non-small cell lung, colorectal and prostate cancer [29]. To analyze the function of pLLO-hEGF in anti-tumor therapy, six cancer cells were selected: HCT116, HT-29, A549, NCI-H157, MDA-MB-231 and SK-BR-3.

\section{The effect of pLLO-hEGF on cancer cell proliferation}

In most cases, EGFR mutations are involved in the development of tumor resistance against traditional EGFR-targeted drugs, and can lead to proliferation signaling of cancer cells independent of EGF binding $[13,14]$. The precondition of pLLO-hEGF applied in antitumor therapy is that it cannot accelerate cancer cell proliferation due to its "EGF tail". Therefore, the growth curve of different cancer cells under different concentrations of pLLOhEGF from low to high was examined (Fig. 3A). For closer investigation, the effects of EGF on cancer cells under the same concentrations with pLLO-hEGF were also monitored (Fig. 3B). The results showed that under different concentrations of pLLO-hEGF or EGF, cancer cells showed nearly the same growth curves (no significant difference, $P>0.05$ ) and the effects resembled those with PBS alone (Fig. 3). 
Fig. 1. Design, expression, purification and identification of recombinant protein pLLO-hEGF. A Design of recombinant protein pLLO-hEGF containing three immunodominant epitopes LL091-99, LL0189-201 and LLO215-226 as well as human EGF sequence and the 6 amino acid linker "GSGGSG". Restriction enzyme sites (BamH I and Xho I) are indicated with black arrows. B Overexpression of pLLO-hEGF in the form of inclusion bodies induced by IPTG at $37^{\circ} \mathrm{C}$ (black arrows). Lane $M$, molecular weight marker. Lane 1 , bacteria transformed with pET-30a(+) without IPTG induction. Lane 2 , bacteria transformed with pET$30 \mathrm{a}(+)$ with $1.0 \mathrm{mM}$ IPTG induc-

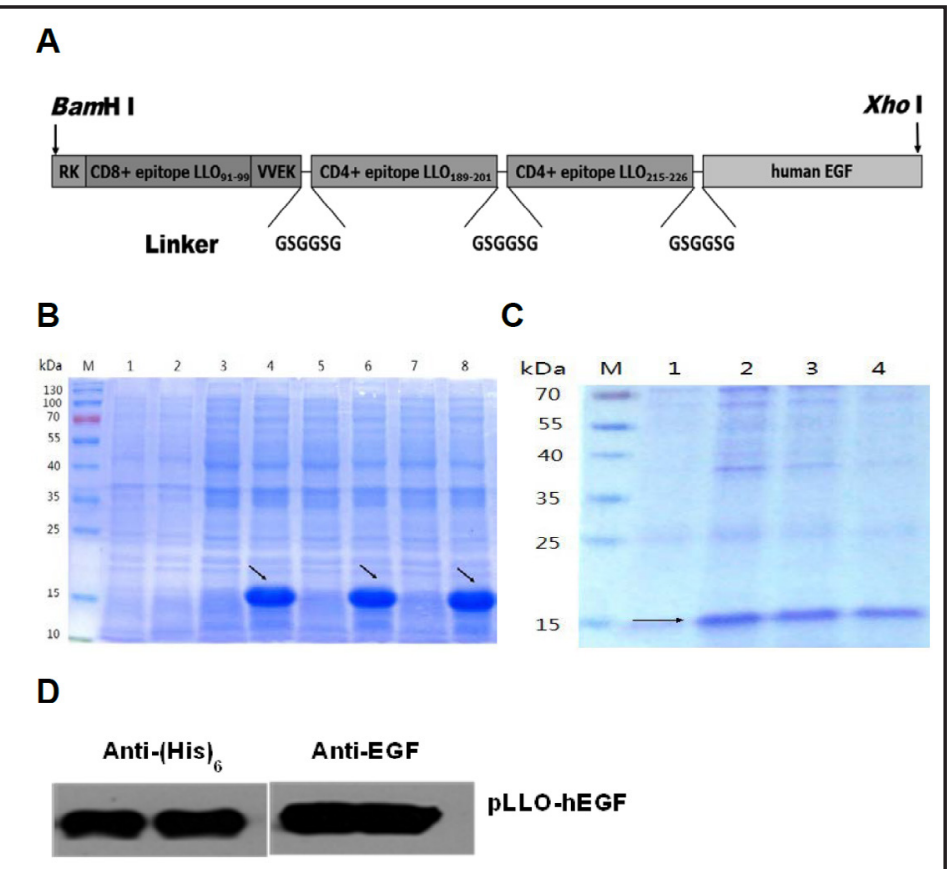

tion. Lane 3, 5 and 7, bacteria transformed with pET-30a(+)-pLLO-hEGF without IPTG induction. Lane 4, 6 and 8 , bacteria transformed with pET-30a(+)-pLLO-hEGF with $1.0 \mathrm{mM}$ IPTG induction. C Purification of pLLO-hEGF in the form of soluble protein using affinity chromatography (black arrow). Lane $M$, molecular weight marker. Lane 1-4, eluate from Co2+ chromatographic column. D Verification of pLLO-hEGF by western blot assay using anti-(His) $6 \mathrm{mAb}$ before thrombin-cleavage of the $6 \times$ His tag and anti-EGF mAb after removing the $6 \times$ His tag.

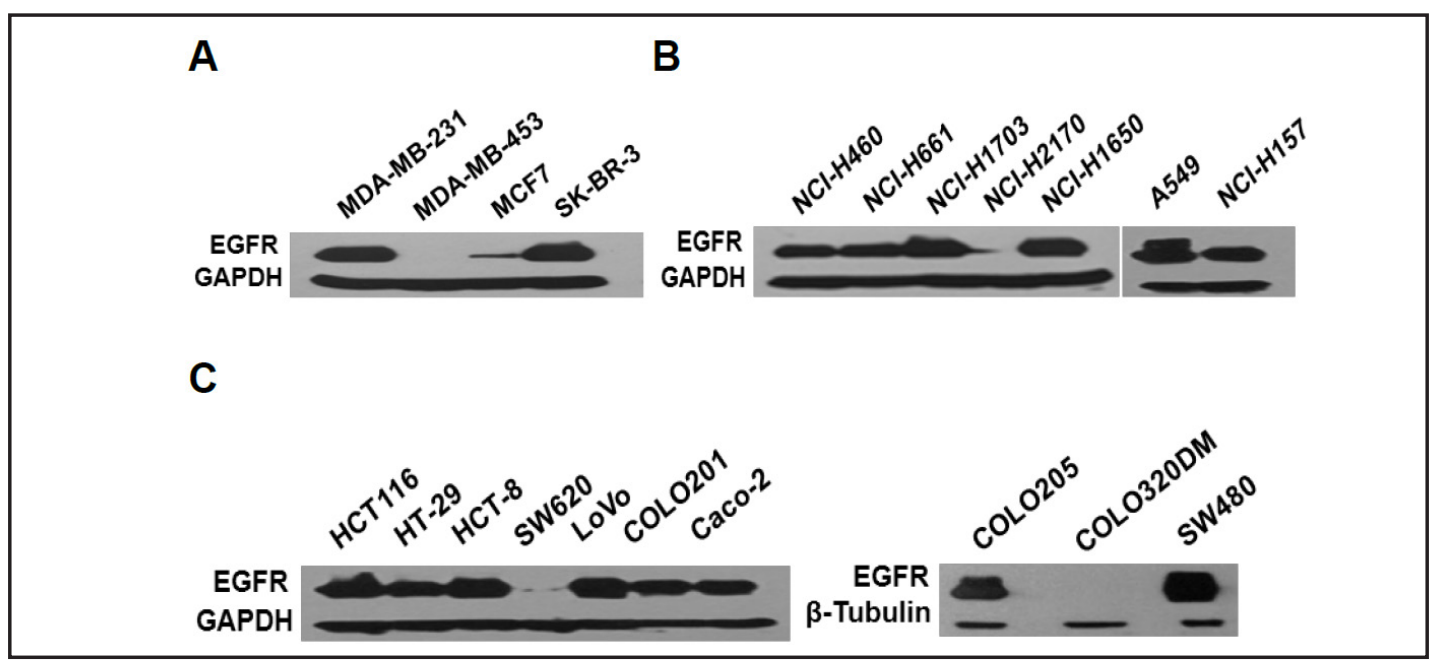

Fig. 2. Expression of EGFR in different human cancer cell lines. A Western blot analysis of EGFR in human breast cancer cell lines including MDA-MB-231, MDA-MB-453, MCF7 and SK-BR-3 (GAPDH as internal reference). B Western blot analysis of EGFR in human lung cancer cell lines including NCI-H460, NCI-H661, NCI-H1703, NCI-H2170, NCI-H1650, A549 and NCI-H157 (GAPDH as internal reference). C Western blot analysis of EGFR in human colorectal cancer cell lines including HCT116, HT-29, HCT-8, SW620, LoVo, COLO201, Caco-2, COLO205, COLO320DM and SW480 (GAPDH and $\beta$-Tubulin as internal references).

pLLO-hEGF can bind to EGFR-expressing cancer cell effectively

Immunocytochemistry was used to determine if pLLO-hEGF could bind to EGFRexpressing cancer cells. Anti-EGFR mAb and protein EGF were used as parallel controls. 
Fig. 3. Growth curves of cancer cells under different concentrations of EGF and pLLO-hEGF by MTT assay. Both EGF and pLLO-hEGF were set the same concentration gradients as follows: $10-10 \mathrm{M}, 10-9$ $\mathrm{M}, 10-8 \mathrm{M}$, and 10-7 M, with PBS as the blank control. A, B The effects of pLLO-hEGF or EGF on proliferation of human cancer cells including HCT116, HT-29, A549, NCI-H157, SKBR-3 and MDA-MB-231. 10-10 M, 10-9 M, 10-8 $\mathrm{M}$, and $10-7 \mathrm{M}$ are indicated as [-10], [-9], [-8] and [-7] respectively; $n$ $=3$ independent experiments.
A
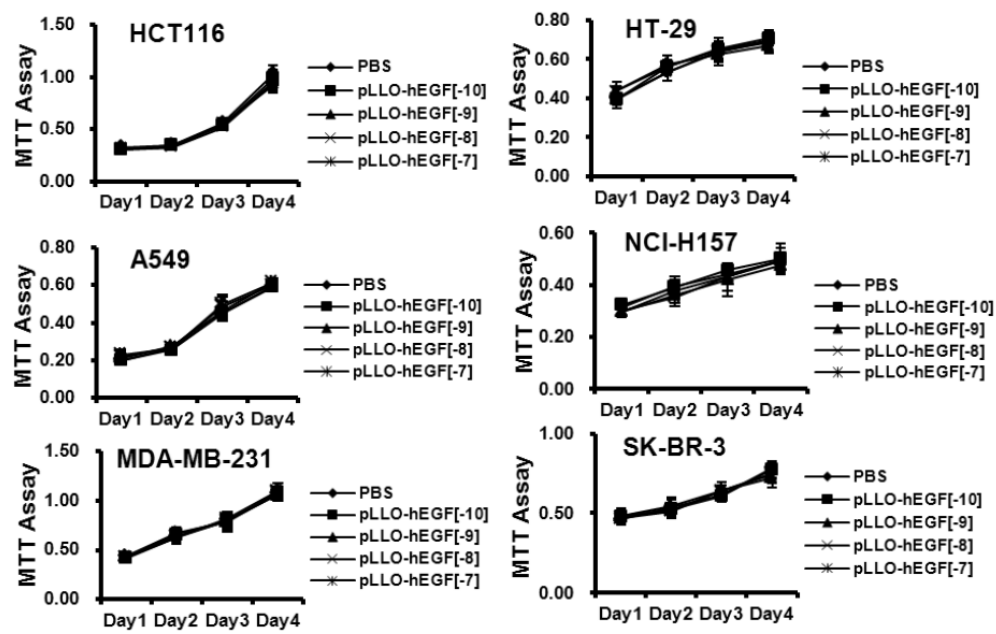

B
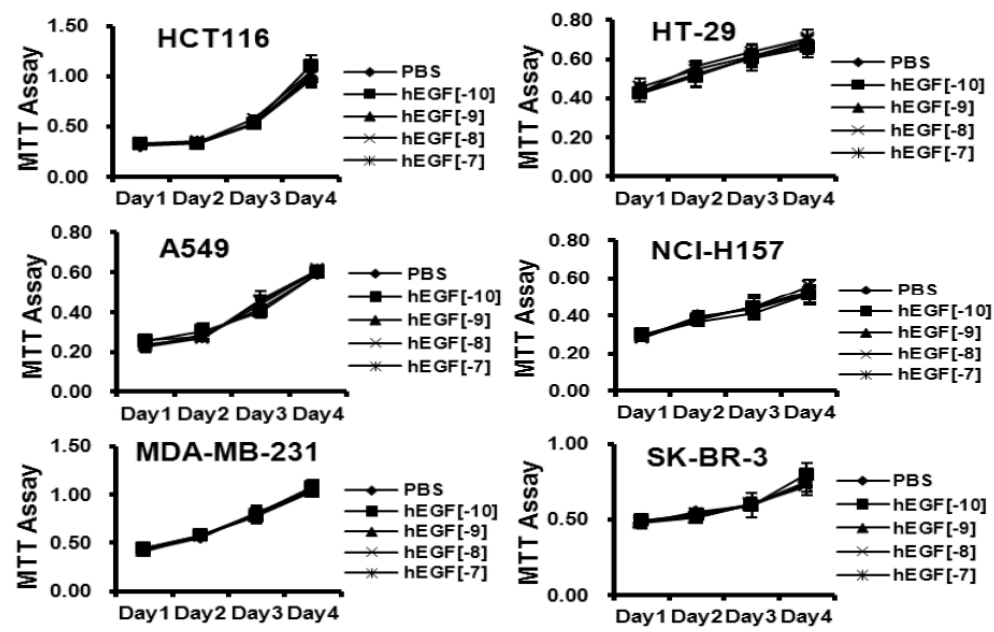

Cancer cells were cultured on slides and fixed by $4 \%$ paraformaldehyde. DAPI coloration was used to visualize the cellular nucleus and the binding of anti-EGFR mAb, EGF and pLLOhEGF to cell surface EGFR was determined using FITC-tagged secondary antibodies (Fig. 4). Compared with PBS group (negative results and data not shown), fluorescent images revealed that like anti-EGFR mAb and EGF, pLLO-hEGF could successfully bind to EGFRexpressing cancer cells (Fig. 4).

\section{pLLO-hEGF can stimulate human T cell proliferation}

Lymphocyte proliferation assays were performed to analyze the immunogenicity of pLLO-hEGF. Human PBMCs were stimulated by pLLO-hEGF, Con A and PBS. A representative result on day 14 , showed that compared to PBS group $\left(44.8 \% \mathrm{CD}^{+} \mathrm{CD} 4^{+}, 32.0 \% \mathrm{CD}^{+} \mathrm{CD}^{+}\right)$, pLLO-hEGF group had a higher proportion of $\mathrm{CD}^{+}{ }^{+} \mathrm{CD} 4^{+} \mathrm{T}$ cells $(54.5 \%)$ and $\mathrm{CD}^{+}{ }^{+} \mathrm{CD} 8^{+} \mathrm{T}$ cells (34.1\%), while Con A group had a low proportion of $\mathrm{CD} 3{ }^{+} \mathrm{CD} 4^{+} \mathrm{T}$ cells and a high ratio of $\mathrm{CD}^{+}{ }^{+} \mathrm{CD} 8^{+} \mathrm{T}$ cells $\left(13.6 \% \mathrm{CD}^{+} \mathrm{CD}^{+}, 69.7 \% \mathrm{CD}^{+} \mathrm{CD}^{+}\right)$(Fig. $\left.5 \mathrm{~A}\right)$. Statistical results revealed that compared with the PBS group there was no significant increasing trend of the numbers of $\mathrm{CD}^{+}, \mathrm{CD}^{+} \mathrm{CD}^{+}$and $\mathrm{CD}^{+}{ }^{+} \mathrm{CD} 8^{+} \mathrm{T}$ cells from day 3 to day 14 ; in the pLLO-hEGF group, the numbers of $\mathrm{CD}^{+}$and $\mathrm{CD}^{+} \mathrm{CD} 4^{+} \mathrm{T}$ cells on day 14 were significantly increased compared 
Fig. 4. pLLO-hEGF binding to different EGFR-expressing cancer cells. Anti-EGFR mAb and EGF were used as the parallel controls. Representative fluorescent images for different groups binding to colorectal cancer cells (HCT116 and HT-29) (A), lung cancer cells (A549 and NCI-H157) (B), and breast cancer cells (MDA-MB-231 and SKBR-3) (C) at $200 \times$ magnification. $D$, anti-EGFR mAb group. E, EGF group. F, pLLO-hEGF group. i: BF, ii: DAPI, iii: FITC.

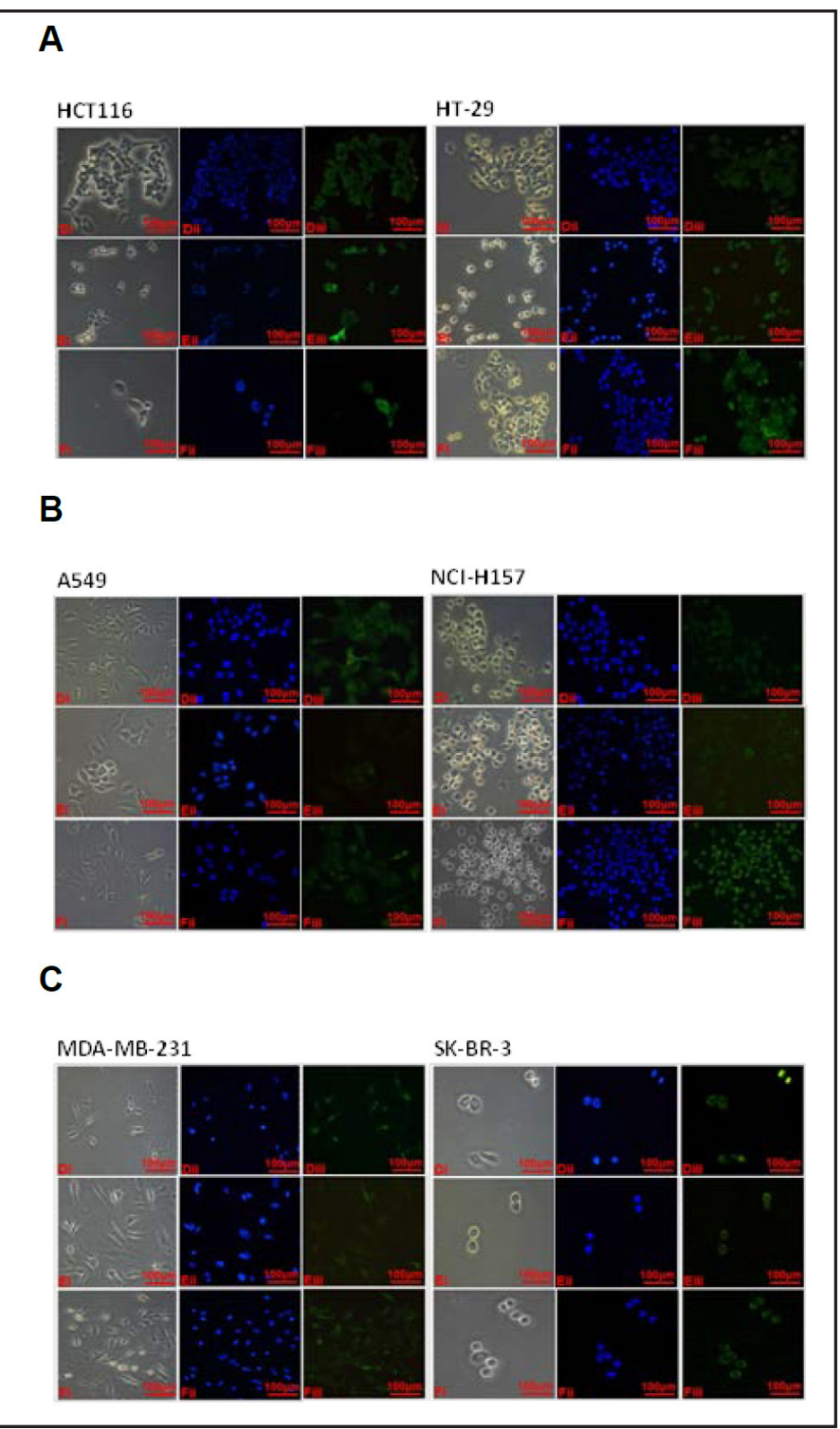

to day 3, but no increase was observed in $\mathrm{CD}^{+} \mathrm{CD}^{+} \mathrm{T}$ cells (Fig. 5B). Conversely, Con $\mathrm{A}$ group showed a significant increase of $\mathrm{CD}^{+} \mathrm{CD}^{+} \mathrm{T}$ cells (Fig. 5B). Previous studies showed that Con A can activate human PBMCs and promote CTL (CD8 ${ }^{+}$T cell) differentiation and development [30, 31].

MTT-based cytotoxicity assay results revealed that under the 50:1 E:T ratio, compared to PBS, cancer cells could be killed efficaciously by lymphocytes primed by pLLO-hEGF in the present of pLLO-hEGF $(P<0.05)$ (Fig. 5C). The lymphocytes stimulated by Con A also showed enhanced killing effects compared with PBS $(P<0.05)$ (Fig. 5C). The percentages of cell lysis for different cancer cells are as follows: HCT116, pLLO-hEGF [(69.89 \pm 1.05$) \%]$

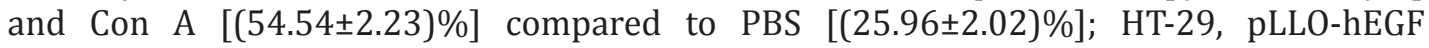

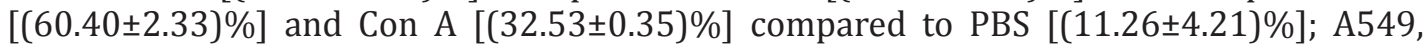

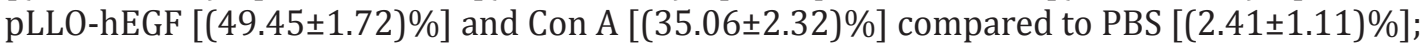
NCI-H157, pLLO-hEGF $[(85.26 \pm 4.82) \%]$ and Con A [(79.49 \pm 1.21$) \%]$ compared to PBS

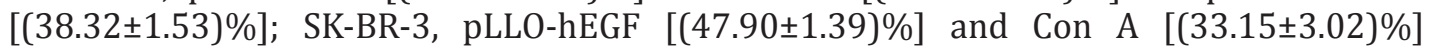

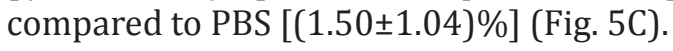

\section{KARGER}




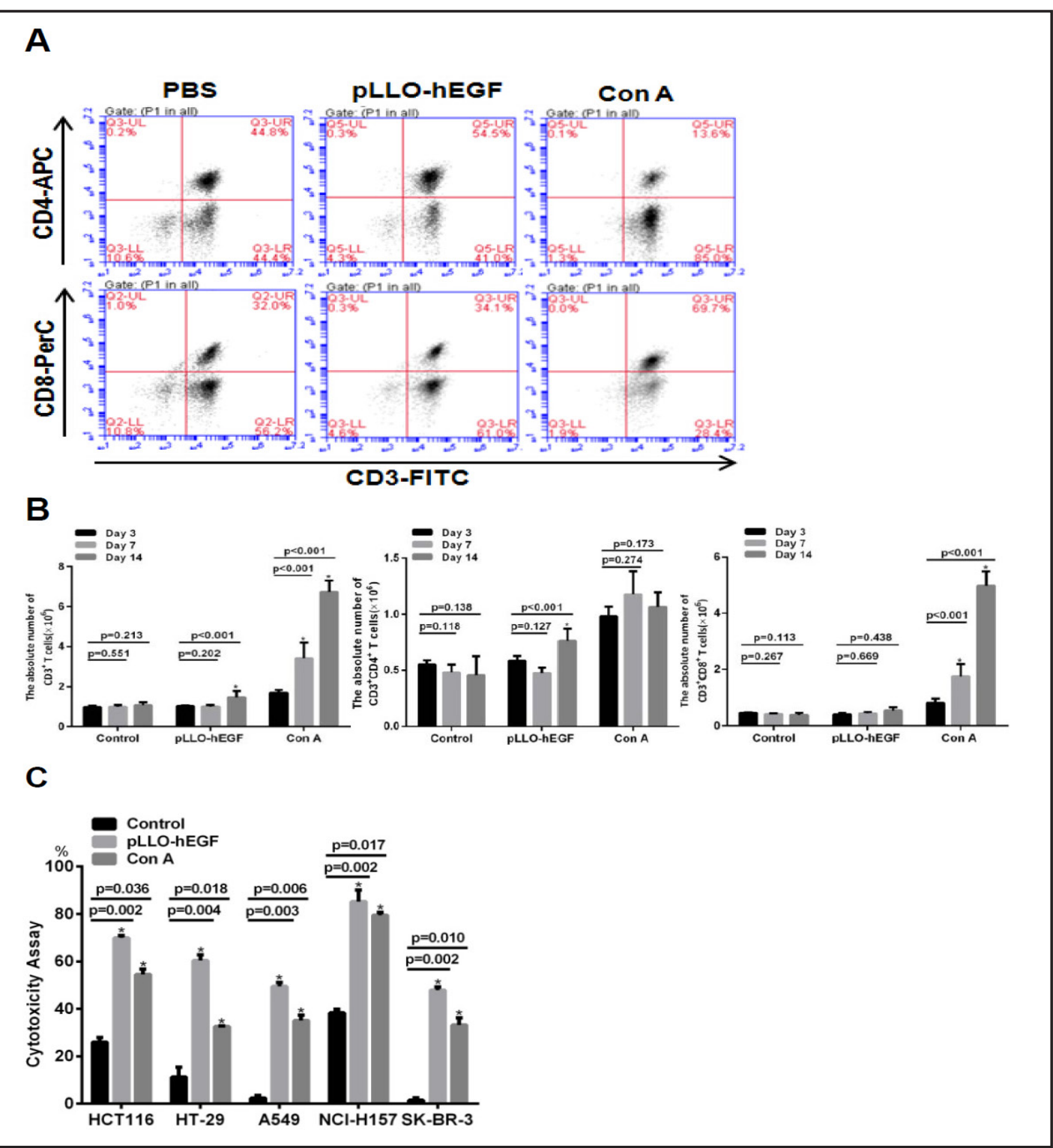

Fig. 5. Antigenicity analysis of pLLO-hEGF by lymphocytes proliferation and cytotoxicity assay. Human PBMCs were divided into three groups: PBS (control), pLLO-hEGF $(10 \mu \mathrm{g} / \mathrm{ml}$ ) and Con A (5 $\mu \mathrm{g} / \mathrm{ml}$ ) for a 14 day culture. A Flow cytometric results of CD3+, CD3+CD4+ and CD3+CD8+ T cells for each group on day 14. B The absolute numbers of CD3+, CD3+CD4+ and CD3+CD8+ T cells for three groups on day 3,7 and $14\left(^{*} P<\right.$ 0.05 relative to day 3 values). C Killing rates in different cancer cells (HCT116, HT-29, A549, NCI-H157 and SK-BR-3) by lymphocytes based on MTT assay. ${ }^{*} P<0.05$, compared with the PBS group; $\mathrm{n}=3$ independent experiments.

\section{Lymphocytes primed by $p L L O-h E G F$ show enhanced anti-tumor activity in vivo}

The anti-tumor activity of lymphocytes primed by pLLO-hEGF was evaluated using a transplanted tumor model (Fig. 6A). There was a significant increase of body weight of mice in Group B and Group D at day 25 compared with day $0(P<0.05)$ that proved no bio-toxicity of pLLO-hEGF (Fig. 6B). In addition, there was a significant decrease of tumor size for Group $\mathrm{B}$ and Group D compared to Group C and Group E, and the average tumor weights were also significantly decreased compared with Group A $(P<0.05)$ (Fig. 6C). Parts of tumor tissues resected from mice were stained with $\mathrm{HE}$ and micrographs were captured under different magnifications. The necrotic area of tumor tissues in Group B and Group D was markedly KARGER 
Fig. 6. Anti-tumor activity of lymphocytes primed by pLLO-hEGF together with pLLO-hEGF in nude mice. Mice were randomly divided into five groups (Groups A-E, see Materials and Methods for more details). A The scheme of anti-tumor therapy in vivo using the HCT116 xenograft model. B The average body weight change of mice each group ( ${ }^{*} P<0.05$ relative to day 0 values). $\mathrm{C}$ The change of average tumor volume and tumor weight of mice each group ${ }^{*} P<0.05$ relative to Group A). D Tumor masses resected from mice, representative HE staining micrographs of each group for necrotic areas and infiltrated lymphocytes $(4 \times 10,20 \times 10,40 \times 10$ magnification respectively) (black arrow), human CD3+ T cells in tumor tissues by immunohistochemistry assay $(20 \times 10$ magnification) (red arrow) and peripheral blood of mice by flow cytometric analysis.

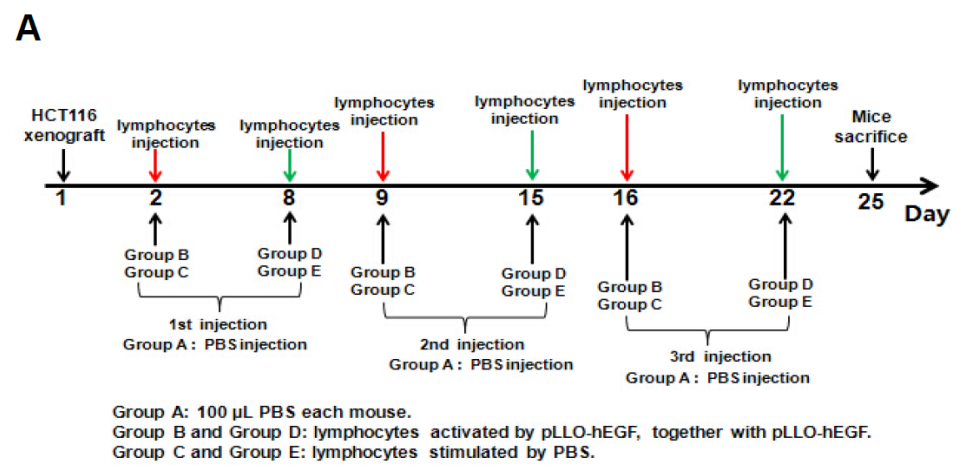

B
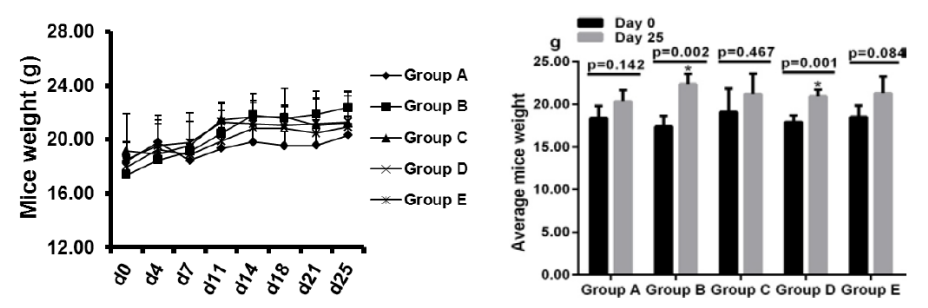

C
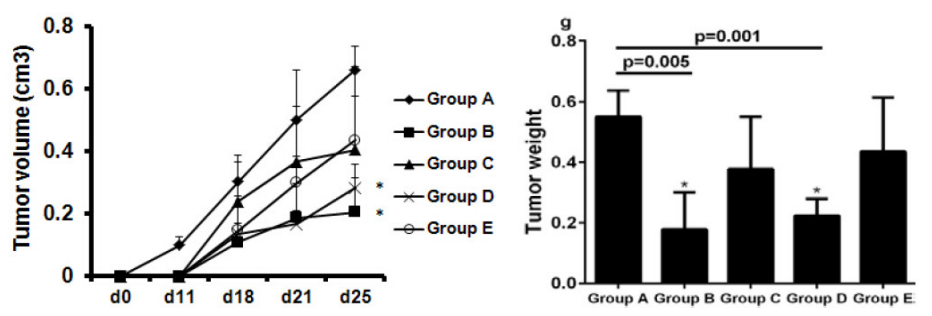

D

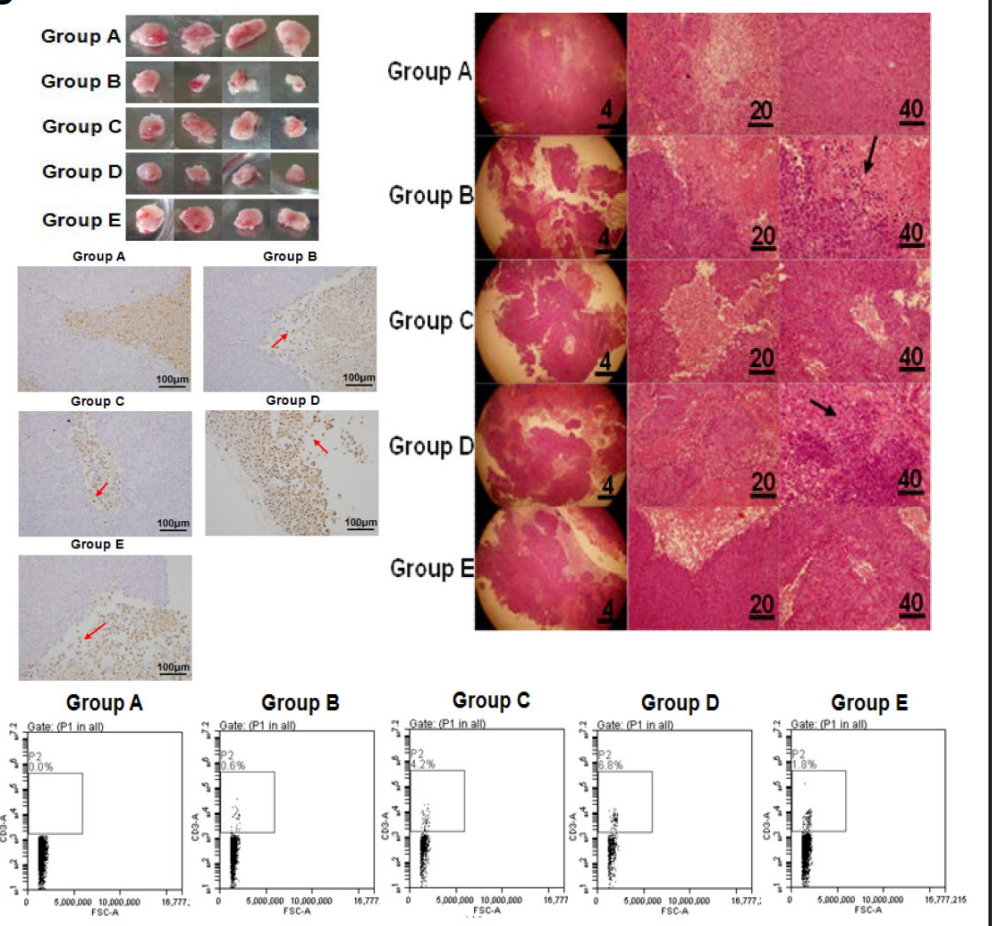


larger than Group A, while Group C and Group E had relatively smaller necrotic areas. In addition, masses of cancer cells turned into lytic necrosis in Group B and Group D, and surrounded by accumulated lymphocytes (Fig. 6D). Immunohistochemistry assay proved that the majority of infiltrated lymphocytes around necrotic areas were injected human lymphocytes (Fig. 6D). Flow cytometric analysis further confirmed that there were human $\mathrm{CD}^{+} \mathrm{T}$ cells in the blood circulation of mice (Fig. 6D). These results suggest that lymphocytes primed by pLLO-hEGF, together with pLLO-hEGF, can lead to significant tumor suppression in mice and reduce the growth rate of tumor xenografts.

\section{Discussion}

The overexpression of EGFR in the majority of cancer cells makes it a well-characterized drug target. At present, mAbs and TKIs are major EGFR-targeted drugs in clinical use for different tumor types. However, most patients do not respond to TKIs or mAbs, and those who do will eventually acquire resistance that typically results from a secondary EGFR mutation [32]. Moreover, there is evidence that EGFR contributes to cancer progression independent of its kinase activity [33]. This suggests that under drug stresses, cancer cells evolve to lose the restriction of EGF-EGFR coupling. Thus, it is necessary to develop new mechanism-based inhibitors or adopt combined therapy measures to prevent or overcome therapeutic resistance in tumors.

The field of cancer immunotherapy has made remarkable progress in the past few years due to improved understanding of the underlying principles of tumor biology and immunology [34]. Currently, cancer immunotherapy faces certain challenges, including weak immunogenicity of tumor cells and immunosuppressive mechanisms, which limit its efficacy. The use of bacteria or bacteria-derived molecules (such as lipid A) has enabled great progress in therapeutic anticancer vaccine development, which is a useful approach to break tumor immune tolerance [35, 36]. Bacteria-derived proteins are usually strongly immunogenic and always rich in antigen epitopes to induce immune response, such as LLO [19]. In our study, we reported the first design and expression of the recombinant protein pLLO-hEGF, which is composed of three immunodominant epitopes (peptides) from LLO and human EGF. Given that EGF-EGFR coupling could lose the function of signaling transduction, EGF can be used as a natural target vector for EGFR-targeted therapy. Thus, pLLO-hEGF could serve as a target immunogenic molecule for actively increasing tumor immunogenicity in anti-tumor therapy. One study showed that peptide intra-tumor injection is an effective method to increase tumor cell antigenicity, making tumor cells more antigenic for specific cytotoxic T-lymphocyte attack [37]. Different from peptides that require intra-tumor injection for administration, pLLO-hEGF could target high-level EGFR-expressing cancer cells via its "EGF tail". Peptide-based therapeutics is relatively easily and cheaply synthesized using recombinant or chemical synthesis techniques. Papo's group designed a new hybrid peptide (D-K6L9) that showed obvious cytotoxicity against TKI-resistant cancer cells [38]. In this study, we generated recombinant protein pLLO-hEGF and through induction condition exploration amounts of soluble form of pLLO-hEGF were acquired successfully. By cell immunofluorescent staining assay, we found that pLLO-hEGF retained the capacity of EGF binding and could bind to EGFR-expressing cancer cells successfully. We then selected representative cancer cell lines (breast, lung and colorectal cancers) to detect if pLLO-hEGF could accelerate cell proliferation. Regardless of the concentration (from low to high) of pLLO-hEGF, the growth curves of cancer cells were not changed. This phenomenon could be favorable for pLLO-hEGF application in anti-tumor therapy. Compared with PBS or Con A, we confirmed that pLLO-hEGF could stimulate $\mathrm{CD} 3^{+} \mathrm{CD} 4^{+} \mathrm{T}$ cell proliferation that was very important to activate antigen-specific immune response. One possible question is why this effect was observed when the concentration of pLLO-hEGF was low. However, one early study has proven that antigen-presenting cells (APCs) pulsed with antigen in the presence of PDGF or EGF are able to stimulate antigen-specific T-cell proliferation to a greater extent 
than APCs pulsed in the absence of exogenous PDGF or EGF [39]. Both growth factors increase the expression of MHC class II antigens on APCs [39]. We initially aimed to use EGF as the vector for supporting immunodominant epitopes to bind the tumor cell surface for enhancing immunogenicity, and now it seems like that the "EGF tail" may facilitate activating the antigen-specific immune response. We also demonstrated that cancer cells could be effectively killed by lymphocytes activated by pLLO-hEGF when they bound to pLLO-hEGF in vitro. Finally, using a transplanted tumor model, we found that lymphocytes activated by pLLO-hEGF together with pLLO-hEGF, when infused into tumor-burdened mice, could significantly suppress tumor growth. More importantly, there was no evident bio-toxic reaction when pLLO-hEGF was injected into mice. Moreover, the injected pLLO-hEGF as antigens also contributed the survival of antigen-specific human lymphocytes in nude mice, which was confirmed by immunohistochemistry and flow cytometric analysis.

In our study, we preliminarily analyzed the function of lymphocytes activated by pLLOhEGF to kill cancer cells in vitro and in vivo. However, the application of pLLO-hEGF in antitumor therapy may have greater potential. For instance, pLLO-hEGF can be used to prepare inactivated tumor cell vaccines. In the future, personalized cancer medicine based on molecular profiling of tumors will be the basis of treatment strategies. Clear understanding of intra- and inter-EGFR signaling pathway connections is essential and proper selection of patients for targeted agents is mandatory. Similar to pLLO-hEGF, the targeted therapy is specifically directed at patients with EGF-EGFR function loss. This study can provide a new direction for future EGFR-targeted drug development.

\section{Acknowledgments}

We thank Beijing Red Cross Blood Center for providing fresh blood samples from healthy donors. This work was supported by the National Science and Technology Infrastructure Grant, China, and has been granted an application number for a patent (No.: 201410152549.2) by the State Intellectual Property office of the P.R.C.

\section{Disclosure Statement}

The authors declare they have no conflict of interest and no financial interest with the subject matter or materials discussed in the manuscript.

\section{References}

1 Verbeek BS, Adriaansen-Slot SS, Vroom TM, Beckers T, Rijksen G: Overexpression of EGFR and c-erbB2 causes enhanced cell migration in human breast cancer cells and NIH3T3 fibroblasts. FEBS Lett 1998 425:145-150.

-2 Singh RK, Lokeshwar BL: The IL-8-regulated chemokine receptor CXCR7 stimulates EGFR signaling to promote prostate cancer growth. Cancer Res 2011;71:3268-3277.

-3 Badalian G, Derecskei K, Szendroi A, Szendroi M, Timar J: EGFR and VEGFR2 protein expressions in bone metastases of clear cell renal cancer. Anticancer Res 2007;27:889-894.

4 Chang L, Shi R, Yang T, Li F, Li G, Guo Y, Lang B, Yang W, Zhuang Q, Xu H: Restoration of LRIG1 suppresses bladder cancer cell growth by directly targeting EGFR activity. J Exp Clin Cancer Res 2013;32:101.

-5 Owens OJ, Stewart C, Brown I, Leake RE: Epidermal growth factor receptors (EGFR) in human ovarian cancer. Br J Cancer 1991;64:907-910.

6 Overman MJ, Hoff PM: EGFR-targeted therapies in colorectal cancer. Dis Colon Rectum 2007;50:12591270. 


\section{Cellular Physiology Cell Physiol Biochem 2015;35:1877-1891 \begin{tabular}{l|l|l}
\hline DOI: $10.1159 / 000373998$ & C 2015 S. Karger AG, Basel
\end{tabular}

Sun et al.: EGFR-Targeted Tumor Biotherapy by a Recombinant Protein

7 Barker FG, 2nd, Simmons ML, Chang SM, Prados MD, Larson DA, Sneed PK, Wara WM, Berger MS, Chen P, Israel MA, Aldape KD: EGFR overexpression and radiation response in glioblastoma multiforme. Int J Radiat Oncol Biol Phys 2001;51:410-418.

8 Kawai H, Ishii A, Washiya K, Konno T, Kon H, Yamaya C, Ono I, Ogawa J: Combined overexpression of EGFR and estrogen receptor alpha correlates with a poor outcome in lung cancer. Anticancer Res 2005;25:46934698.

9 Maiti GP, Mondal P, Mukherjee N, Ghosh A, Ghosh S, Dey S, Chakrabarty J, Roy A, Biswas J, Roychoudhury S, Panda CK: Overexpression of EGFR in Head and Neck squamous cell carcinoma is associated with inactivation of SH3GL2 and CDC25A genes. Plos One 2013;8.

10 Troiani T, Martinelli E, Capasso A, Morgillo F, Orditura M, De Vita F, Ciardiello F: Targeting EGFR in pancreatic cancer treatment. Curr Drug Targets 2012;13:802-810.

11 Chong CR, Janne PA: The quest to overcome resistance to EGFR-targeted therapies in cancer. Nat Med 2013;19:1389-1400.

12 Gomez GG, Wykosky J, Zanca C, Furnari FB, Cavenee WK: Therapeutic resistance in cancer: microRNA regulation of EGFR signaling networks. Cancer Biol Med 2013;10:192-205.

13 Yun CH, Boggon TJ, Li Y, Woo MS, Greulich H, Meyerson M, Eck MJ: Structures of lung cancer-derived EGFR mutants and inhibitor complexes: mechanism of activation and insights into differential inhibitor sensitivity. Cancer Cell 2007;11:217-227.

14 Hrustanovic G, Lee BJ, Bivona TG: Mechanisms of resistance to EGFR targeted therapies. Cancer Biol Ther 2013;14:304-314.

15 Blankenstein T, Coulie PG, Gilboa E, Jaffee EM: The determinants of tumour immunogenicity. Nature Revi Cancer 2012;12:307-313.

16 Nauts HC, McLaren JR: Coley toxins--the first century. Adv Exp Med Biol 1990;267:483-500.

17 Schnupf P, Zhou J, Varshavsky A, Portnoy DA: Listeriolysin O secreted by Listeria monocytogenes into the host cell cytosol is degraded by the N-end rule pathway. Infect Immun 2007;75: 5135-5147.

18 Pamer EG, Harty JT, Bevan MJ: Precise prediction of a dominant class I MHC-restricted epitope of Listeria monocytogenes. Nature 1991;353:852-855.

19 Verma NK, Ziegler HK, Wilson M, Khan M, Safley S, Stocker BA, Schoolnik GK: Delivery of class I and class II MHC-restricted T-cell epitopes of listeriolysin of Listeria monocytogenes by attenuated Salmonella. Vaccine 1995;13:142-150.

20 Rodriguez-Del Rio E, Frande-Cabanes E, Tobes R, Pareja E, Lecea-Cuello MJ, Ruiz-Sáez M, Carrasco-Marín E, Alvarez-Dominguez C: The intact structural form of LLO in endosomes cannot protect against listeriosis. Int J Biochem Mol Biol 2011; 2: 207-218.

21 Kayal S, Charbit A: Listeriolysin 0: a key protein of Listeria monocytogenes with multiple functions. FEMS Microbiol Rev 2006;30:514-529.

22 Lety MA, Frehel C, Berche P, Charbit A: Critical role of the N-terminal residues of listeriolysin 0 in phagosomal escape and virulence of Listeria monocytogenes. Mol Microbiol 2002;46:367-379.

23 Wang L, Yao J, Shi X, Hu L, Li Z, Song T, Huang C: MicroRNA-302b suppresses cell proliferation by targeting EGFR in human hepatocellular carcinoma SMMC-7721 cells. BMC Cancer 2013;13:448.

-24 Wood SA, Kaye PL: Effects of epidermal growth factor on preimplantation mouse embryos. J Reprod Fertil 1989;85:575-582.

25 Li Y, Fan J, Chen M, Li W, Woodley DT: Transforming growth factor-alpha: a major human serum factor that promotes human keratinocyte migration. J Invest Dermatol 2006;126:2096-2105.

26 Lee JS, Latimer LJ, Burkholder GD: Immunofluorescent staining of chromosomes with DNA-binding antibodies. Methods Mol Biol 1997;90:189-193.

27 Plumb JA, Milroy R, Kaye SB: Effects of the pH dependence of 3-(4,5-dimethylthiazol-2-yl)-2,5-diphenyltetrazolium bromide-formazan absorption on chemosensitivity determined by a novel tetrazolium-based assay. Cancer Res 1989;49:4435-4440.

-28 Feller JK, Yang S, Mahalingam M: Immunohistochemistry with a mutation-specific monoclonal antibody as a screening tool for the BRAFV600E mutational status in primary cutaneous malignant melanoma. Modern Pathology 2013;26:414-420.

29 Salomon DS, Brandt R, Ciardiello F, Normanno N: Epidermal growth factor-related peptides and their receptors in human malignancies. Critical Rev Oncol Hematol 1995;19:183-232. 


\section{Cellular Physiology Cell Physiol Biochem 2015;35:1877-1891

and Biochemistry

-30 Rosloniec EF, Cleveland RP, Kaufman DB: Induction of T-cell proliferation and enhancement of Nk activity by supernatants from Con a-stimulated human peripheral-blood mononuclear-cells - a new lymphokine. Cell Immunol 1986;99:170-181.

-31 Falkoff RM, Dutton RW: Evidence that Con A induces cytotoxicity in the same subclass of T cells as does alloimmunization. J Immunol 1977;118:1600-1606.

-32 Giaccone G, Wang Y: Strategies for overcoming resistance to EGFR family tyrosine kinase inhibitors. Cancer Treat Rev 2011;37:456-464.

-33 Weihua Z, Tsan R, Huang WC, Wu Q Chiu CH, Fidler IJ, Hung MC: Survival of cancer cells is maintained by EGFR independent of its kinase activity. Cancer Cell 2008;13:385-393.

-34 Kirkwood JM, Butterfield LH, Tarhini AA, Zarour H, Kalinski P, Ferrone S: Immunotherapy of cancer in 2012. CA Cancer J Clin 2012;62:309-335.

-35 Shahabi V, Maciag PC, Rivera S, Wallecha A: Live, attenuated strains of Listeria and Salmonella as vaccine vectors in cancer treatment. Bioeng Bugs 2010;1:235-243.

-36 Reisser D, Pance A, Jeannin JF: Mechanisms of the antitumoral effect of lipid A. Bioessays 2002;24:284-289.

37 Nobuoka D, Yoshikawa T, Fujiwara T, Nakatsura T: Peptide intra-tumor injection for cancer immunotherapy: enhancement of tumor cell antigenicity is a novel and attractive strategy. Hum Vaccin Immunother 2013;9:1234-1236.

-38 Papo N, Seger D, Makovitzki A, Kalchenko V, Eshhar Z, Degani H, Shai Y: Inhibition of tumor growth and elimination of multiple metastases in human prostate and breast xenografts by systemic inoculation of a host defense-like lytic peptide. Cancer Res 2006;66:5371-5378.

-39 Acres RB, Lamb JR, Feldman M: Effects of platelet-derived growth factor and epidermal growth factor on antigen-induced proliferation of human T-cell lines. Immunology 1985;54:9-16. 